\title{
CORRECTION
}

\section{Correction to: Stabilization of the Q-switching pulse energy of a composite YAG/Yb:YAG/Cr:YAG laser used as a laser ignitor}

\author{
Youngin Park ${ }^{1}$. Jeonghyun Chang ${ }^{1} \cdot$ Seongsoo Kim ${ }^{1} \cdot$ Hyun Su Kim ${ }^{1}$
}

Published online: 19 May 2021

(c) The Korean Physical Society 2021

Correction to: Journal of the Korean Physical Society https://doi.org/10.1007/s40042-021-00103-4

Due to an unfortunate oversight this paper was published with typographical errors in Keywords and Eq. (5).

In keywords, Yb · YAG should be changed to one keyword, Yb:YAG.

In Eq. (5), $h v_{L}$ should be changed to 1 as follows:

$\frac{\phi_{\mathrm{ASE}}}{t_{r}}=\frac{\Omega}{4 \cdot t_{r} \cdot c} \cdot \frac{1}{\tau_{g} \sigma_{g}} \cdot \frac{G_{0}}{\left(\ln G_{0}\right)^{1 / 2}}$.

1 Department of Photonic Engineering, Chosun University, Gwangju 61452, Republic of Korea 